\title{
Multiple watermarking technique using optimal threshold
}

\author{
Noruhida Alias, Ferda Ernawan \\ Faculty of Computer Systems \& Software Engineering, Universiti Malaysia Pahang, Malaysia
}

\begin{tabular}{l} 
Article Info \\
\hline Article history: \\
Received Aug 19, 2019 \\
Revised Oct 21, 2019 \\
Accepted Nov 5, 2019 \\
\hline Keywords: \\
Arnold transforms \\
Discrete wavelet transform \\
HVS characteristics \\
Multiple watermarking \\
Singular value decomposition
\end{tabular}

\begin{abstract}
The multiple watermarking technique has drawn more attention due to high demand for embedding more than one copyright. This paper proposes a multiple watermarking scheme using DWT-SVD by utilizing HVS characteristics. Our scheme embeds multiple watermarks in red and blue colors. The proposed scheme examines the coefficients of $U$ orthogonal matrix for embedding and extracting watermarks. Watermarks are scrambled by Arnold transform before embedded in the host image in order provide additional security. The optimal thresholds for red and blue colors are revealed by finding a trade-off between normalized-cross correlation and imperceptibility from quantization steps. The experimental results demonstrate that our scheme achieves high resistant under JPEG and JPEG2000 compression for both inserted watermarks.
\end{abstract}

Copyright () 2020 Institute of Advanced Engineering and Science. All rights reserved.

Corresponding Author:

Ferda Ernawan,

Faculty of Computer Systems \& Software Engineering,

Universiti Malaysia Pahang, Malaysia.

Email: ferda@ump.edu.my

\section{INTRODUCTION}

The used of the internet and transferring digital media over the network have led to distribute multimedia data. The usage of the internet allows users to copy and distribute the digital multimedia data. Digital watermarking is an alternative solution to protect the copyright of multimedia data. Digital watermarking has become popular in protecting the copyrights and authentication in the last decade [1-5]. The basic requirements of digital watermarking are its robustness, imperceptibility and security [6].

The multiple watermarking technique can protect multiple ownership of the multimedia data. It also can be used to verify the integrity of the contents. Multiple watermarking embeds more than one watermark image into the host image [7]. Due to rapid technology, multiple watermarks are needed in a variety of applications such as fingerprinting, broadcast monitoring, copyright protection and digital signatures [8-9].

This paper proposes a multiple watermarking scheme using the optimal thresholds on red and blue colors. The color host image is divided into red, green and blue colors. Red and blue color are selected to embed multiple watermarks due to less sensitive to the human visual system (HVS). The HVS entropy is utilized to determine the embedding regions. The smallest entropy value is used to select embedded blocks of the image. Selected blocks are transformed by Discrete Wavelet Transform (DWT) to obtain low-low sub band (LL), high-low sub band (HL), low-high subband (LH) and high-high subband (HH). Singular Value decomposition (SVD) is applied to LL sub band to derive U, S and V matrices. The multiple watermarks are embedded by modifying the first column of $U$ matrix. This paper proposed the optimal thresholds for red and blue colors by examining the imperceptibility and robustness performance against the JPEG quantization step. The optimal thresholds can be achieved as a trade-off between the resistance of extracted watermark and invisible of the watermarked image. 


\section{RELATED WORK}

Researchers [10-13] presented hybrid methods in multiple watermarking scheme. Zear et al. [8] presented multiple watermarking technique based on the hybrid methods in medical images. Their multiple watermarking scheme implements DWT, DCT, SVD and back propagation neural network. Zear's scheme embeds a copyright image and text into the medical image to secure the details of patients. The combination of transforming domain and SVD can improve the robustness and visual quality of the watermarked image. Liu et al. [11] presented a dual watermarking technique for authentication and copyright protection. Author proposed robust and fragile watermarks where the watermarks are embedded into YCbCr and RGB color, respectively. In order to achieve a robustness watermarking scheme, the first watermark is embedded into an LL sub - band of DWT on the luminance channel. In addition, the second watermark is embedded in RGB color for authentication. The combination of robust and fragile watermarking schemes draws more attention to develop multiple watermarking.

Mohananthini and Yamuna [12] discussed multiple watermarking technique using genetic algorithms. Their scheme elaborates multiple watermarking techniques such as segmented, successive and composite watermarking. Their scheme can achieve high robustness when it was compared to a single watermarking technique. Ernawan et al. [7] proposed a blind multiple watermarking technique by considering the human visual characteristics. Ernawan's scheme presented embedding multiple watermarks on luminance $(\mathrm{Y})$ and Chrominance blue $(\mathrm{Cb})$. The converting RGB color to YCbCr may not relevance in multiple watermarking technique. This scheme can provide a good resistance against JPEG compression. Ernawan's scheme [7] embeds two watermarks by modifying the first column of the U matrix of DCT-SVD. Ernawan's scheme can achieve high robustness for both embedded watermarks against signal processing attacks. However, the robustness of the extracted watermark on the luminance against noise attacks still can be improved.

\section{RESEARCH METHOD}

This paper proposes DWT-SVD for the multiple watermarking scheme. A DWT is a multiresolution for a mathematical tool that decompose the image [14-18]. Edges information is presented in the high frequency. Whereas low frequency contains of the visual information [17].

\subsection{SVD}

SVD is a technique of diagonalizable the symmetric matrix in linear algebra [19-20]. SVD is widely applied in digital watermarking due to its good stability and resistant against signal processing attacks [21]. $\mathrm{SVD}$ of an image $\mathrm{A}$ is divided into $\mathrm{U}, \mathrm{S}$ and $\mathrm{V}$ matrices.

$$
A=U S V^{T}
$$

where $\mathrm{S}$ is a diagonal matrix, $\mathrm{U}$ and $\mathrm{V}$ are orthogonal matrices. The elements in matrix $\mathrm{S}$ are called singular values and it is arranged in decreasing order.

\subsection{Arnold Transform}

Arnold transforms is a two-dimensional mapping that changes the coordinate of image pixels [22]. The Arnold transform is used to scramble the watermark image before embedding watermark in the host image. The Arnold transform is given by [23]:

$$
\left(\begin{array}{l}
x^{\prime} \\
y^{\prime}
\end{array}\right)=\left(\begin{array}{ll}
1 & 1 \\
1 & 2
\end{array}\right)\left(\begin{array}{l}
x \\
y
\end{array}\right) \bmod N
$$

where $\left(\begin{array}{l}x \\ y\end{array}\right)$ are the original coordinates and $\left(\begin{array}{l}x^{\prime} \\ y^{\prime}\end{array}\right)$ is the transformed coordinates of image pixels. $\mathrm{N}$ us the number of iterations of scrambled image pixels. Our scheme denotes $\mathrm{N}$ as a private key for scrambling the watermark images.

\subsection{Human Visual System}

The characteristics of the human visual system (HVS) are being utilized to identify the most suitable region to embed the watermarks. HVS characteristics are used to determine the suitable embedding locations and it can avoid significant distortions during the embedding watermark [24]. The entropy of HVS characteristics can be expressed as follows [25]: 


$$
H V S_{E}=\sum_{i=1}^{n} p_{i} \log _{2}\left(p_{i}\right)-p_{i} \exp ^{1-p_{i}}
$$

\section{WATERMARKING SCHEME}

This section explains the proposed multiple watermarking scheme based on DWT-SVD, Arnold transform and the human visual characteristics of red and blue colors. The watermark insertion process is illustrated in the block diagram as shown in Figure 1.

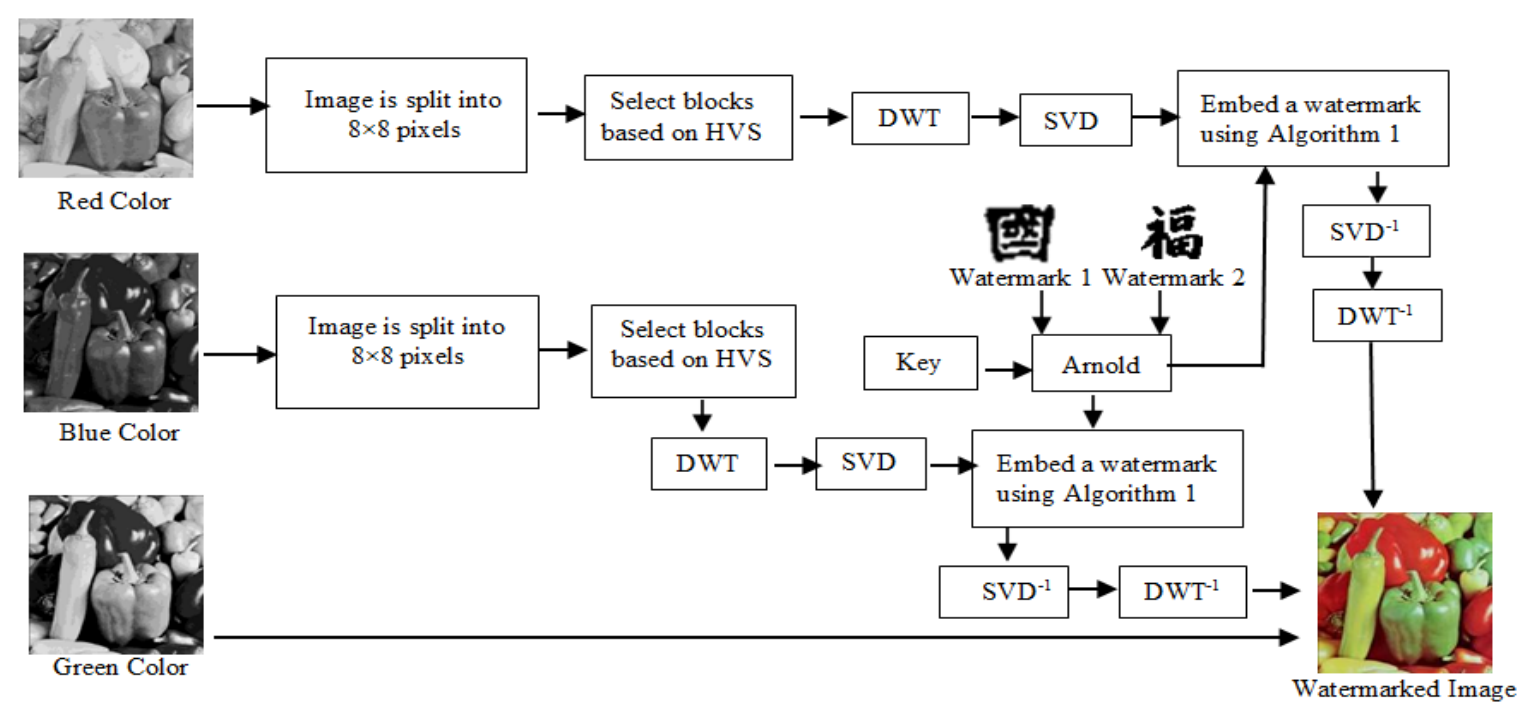

Figure 1. Watermark Insertion

The true color image is decomposed into each red, green and blue color. This experiment selects red and blue colors because it's less sensitive to the human eyes. Red and blue colors are divided into $8 \times 8$ pixels, then it is computed by HVSE as stated in (3). Next, the selected blocks based on HVSE are transformed by DWT, then LL-sub band of DWT is computed by SVD. The selected coefficients on the first column of U matrix are modified based on the optimal thresholds. These thresholds give significant effect to obtain strong resistance of the extracted watermark and invisible watermarked image. The proposed thresholds for red and blue colors are shown in Figure 2.

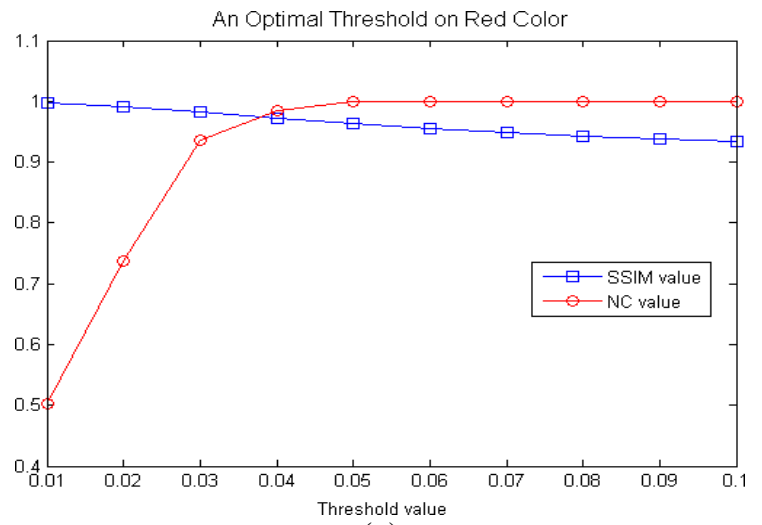

(a)

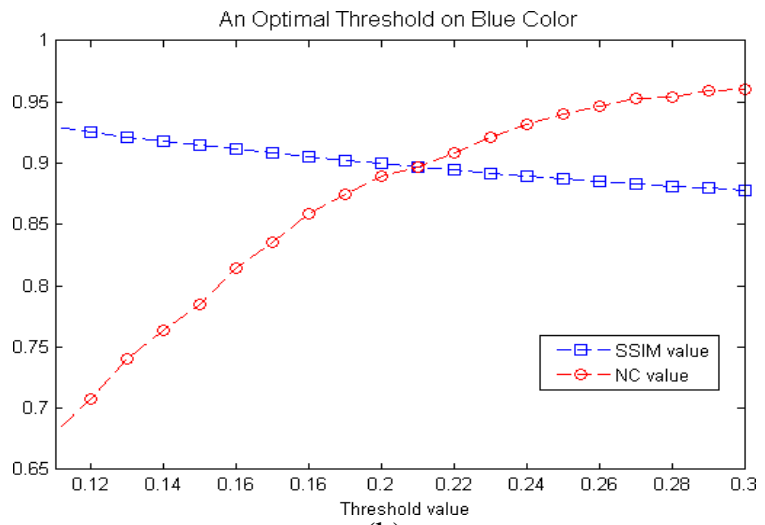

(b)

Figure 2. The proposed threshold on red color (a) and blue color (b) 
This paper proposed threshold values for embedding both watermark on red and blue colors. The thresholds of 0.04 and 0.21 for red and blue colors respectively are proposed for scaling the strength of watermark insertion. These thresholds are obtained from incrementing a threshold once a time of NC and SSIM values against JPEG quantization steps. Referring to Figure 2, these thresholds indicate a trade-off between robustness and imperceptibility of the watermarked image. The NC value indicates the robustness of the extracted watermark after compressed image and SSIM value presents the invisible of the watermarked image before attacks. A trade-off between NC and SSIM indicates the balancing of invisible and resistance of the watermarked image. The watermark insertion and extraction algorithms are elaborated in the next sub-section.

\subsection{Watermark Insertion}

This section discusses the process of watermark insertion in red and blue colors. Our experiments use two binary watermarks with size of $32 \times 32$ pixels and five host images. The watermark insertion process is discussed in Algorithm 1.

\section{Algorithm 1: Insertion}

Input: Host image, two binary watermarks

1. $\quad$ Each block of $8 \times 8$ pixels is computed by $H V S_{E}$ in (3)

2. Select blocks with lowest $H V S_{E}$ value for embedding watermark.

3. Both watermark images are scrambled by Arnold.

4. Apply DWT to the selected blocks in order to obtain LL, LH, HL and HH sub-bands.

5. The LL sub-band of DWT is computed by SVD to obtain $U$ matrix.

6. Each binary watermark is embedded in red and blue colors using the following rules:

Rule 1: Calculate the average of $m=\left(U_{(2,1)}+U_{(3,1)}\right) / 2$

Rule 2: If binary watermark $=1$, the coefficients are modified by:

$$
U_{(2,1)}=x \cdot m+\alpha / 2 \text { where }\left\{\begin{array} { c } 
{ x = 1 , U _ { ( 2 , 1 ) } > 0 } \\
{ x = - 1 , U _ { ( 2 , 1 ) } < 0 }
\end{array} \text { and } U _ { ( 3 , 1 ) } = y \cdot m - \alpha / 2 \text { where } \left\{\begin{array}{c}
y=1, U_{(3,1)}>0 \\
y=-1, U_{(3,1)}<0
\end{array}\right.\right.
$$

Rule 3: If binary watermark $=0$, the coefficients are modified by:

$$
U_{(2,1)}=x \cdot m-\alpha / 2 \text { where }\left\{\begin{array} { c } 
{ x = 1 , U _ { ( 2 , 1 ) } > 0 } \\
{ x = - 1 , U _ { ( 2 , 1 ) } < 0 }
\end{array} \text { and } U _ { ( 3 , 1 ) } = y \cdot m + \alpha / 2 \text { where } \left\{\begin{array}{c}
y=1, U_{(3,1)}>0 \\
y=-1, U_{(3,1)}<0
\end{array}\right.\right.
$$

7. Perform inverse SVD and DWT.

8. Merge all the modified blocks to obtain the watermarked image.

Output: Watermarked image

\subsection{Watermark Extraction}

The step-by-step of extraction process is presented in Algorithm 2. The watermark images are extracted from red and blue colors of host image. The block diagram of the watermark extraction is shown in Figure 3.

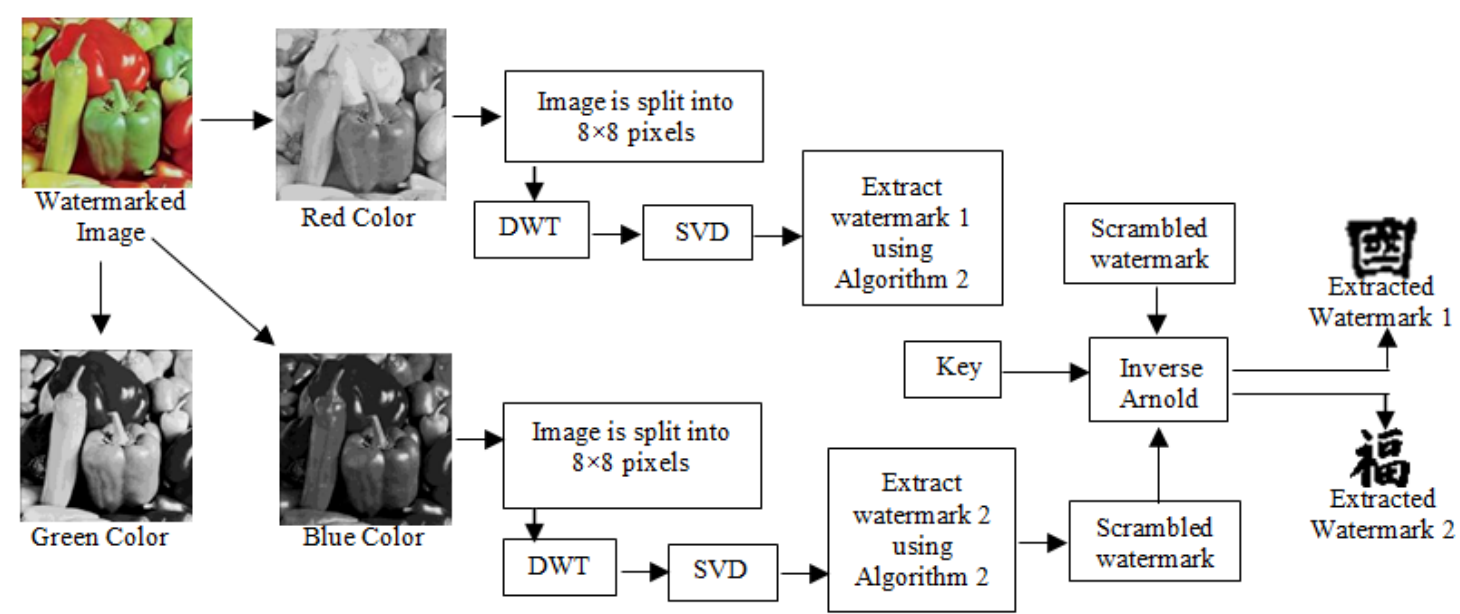

Figure 3. Watermark Extraction 
Algorithm 2: Extraction

1. The watermarked image is decomposed into $8 \times 8$ pixels of non-overlapping blocks.

2. Select the blocks with lowest $\mathrm{HVS}_{\mathrm{E}}$ value, then apply DWT on each selected block.

3. Apply SVD on LL sub-band of DWT.

4. Extract the watermark bits by examining $U_{(2,1)}$ and $U_{(3,1)}$ of DWT-SVD for embedded both watermarks on red and blue colors.

5. Calculate the difference value between $U_{(2,1)}$ and $U_{(3,1)}$ coefficients, if it is greater than 0 , then assign the extracted watermark bit $=1$. Otherwise, the watermark bit $=0$.

6. Perform inverse Arnold transform for extracted both watermarks.

Output: Watermark recovery from red and blue colors.

\section{EXPERIMENTAL RESULTS}

Five host images with size of $512 \times 512$ pixels are identified as Lena, Baboon, Pepper, Sailboat and Airplane as depicted in Figure 4. Two binary watermarks and its scrambled watermarks are shown in Figure 5.

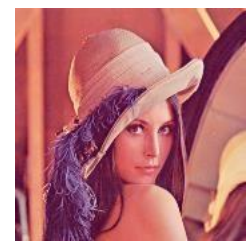

(a)

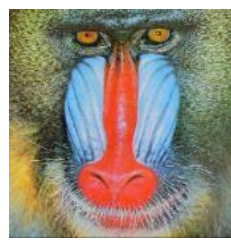

(b)

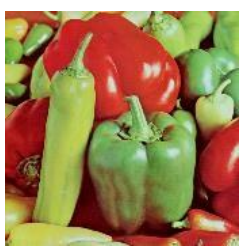

(c)

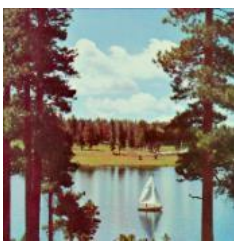

(d)

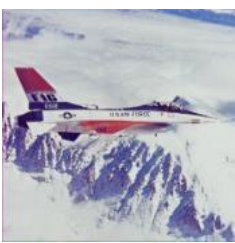

(e)

Figure 4. (a) Lena, (b) Baboon, (c) Pepper, (d) Sailboat, (e) Airplane

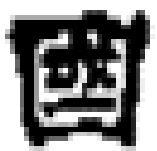

(a)

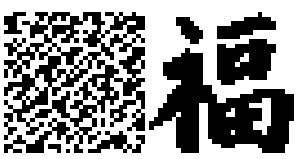

(b) (c)

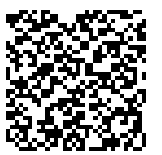

(d)

Figure 5. (a) Watermark 1, (b) Scrambled Watermark 1, (c) Watermark 2, (d) Scrambled Watermark 2

The proposed scheme is tested under various types of attack such as image processing, geometrical attacks and JPEG compression. The performance of the proposed scheme is assessed by Normalized-cross Correlation (NC), Bit Error Rate (BER) and Structural SIMilarity (SSIM) index. The quality of the proposed multiple watermarking scheme is shown in Table 1.

Table 1. Comparison of ARE and SSIM Values between Ernawan's Scheme and Our Scheme

\begin{tabular}{lcccc}
\hline \multirow{2}{*}{ Image } & \multicolumn{2}{c}{ Ernawan [7] } & \multicolumn{2}{c}{ Our scheme } \\
\cline { 2 - 5 } & ARE & SSIM & ARE & SSIM \\
Lena & 1.8774 & 0.8996 & 1.1670 & 0.9083 \\
Baboon & 0.9957 & 0.9482 & 1.3576 & 0.9432 \\
Pepper & 2.7161 & 0.9326 & 0.6556 & 0.9543 \\
Sailboat & 3.8471 & 0.8459 & 1.8012 & 0.9052 \\
Airplane & 3.6957 & 0.8849 & 1.9381 & 0.8675 \\
\hline
\end{tabular}

The highest SSIM value is 1 , it indicates same quality to the original image. The highest ARE represents the highest distortion that appear on the watermarked image. Based on ARE values, it shows that our proposed scheme indicates less distortion. Our scheme also achieves higher imperceptibility than Ernawan's scheme in terms of SSIM values.

The watermarked images of the proposed scheme are shown in Figure 6. The used of DWT-SVD improves the imperceptibility of the watermarked image. It has been proven by obtaining higher SSIM values of the watermarked image than the existing scheme. While, our scheme produces slightly lower SSIM value 
for baboon image due to its texture image. The robustness performance of the proposed scheme is shown in Figure 7. Our scheme achieves high robustness with minimum BER values under JPEG compression with quality factor of 20,30,40, 50 and 60. Embedding multiple watermarks on the color image may be destroyed by JPEG quantization tables. JPEG performs large quantization values in chrominance channels, it may remove the second embedded watermark. While, the proposed scheme shows a great robust performance for both embedded watermarks. The proposed scheme produces minimum BER values for the second extracted watermark image. The proposed scheme is also tested against various image processing and geometrical attacks as shown in Tables 2 and 3.

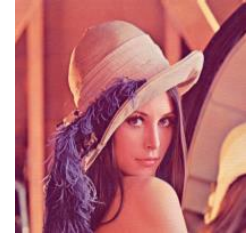

(a)

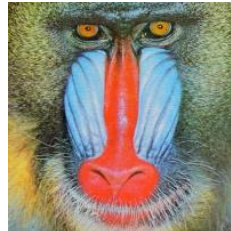

(b)

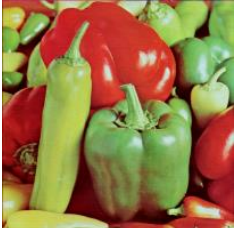

(c)

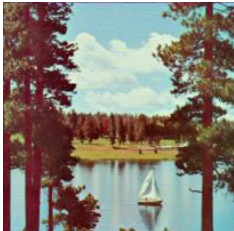

(d)

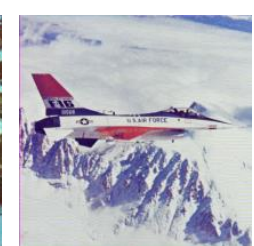

(e)

Figure 6. Watermarked (a) Lena, (b) Baboon, (c) Pepper, (d) Sailboat, (e) Airplane

Table 2. Comparison of NC and BER Values under Image Processing Attacks

\begin{tabular}{ccccccccc}
\hline & \multicolumn{4}{c}{ Watermark 1 } & \multicolumn{5}{c}{ Watermark 2 } \\
\cline { 2 - 11 } Attacks & \multicolumn{2}{c}{ Ernawan [7] } & Proposed Scheme & \multicolumn{2}{c}{ Ernawan [7] } & Proposed Scheme \\
No attacks & NC & BER & NC & BER & NC & BER & NC & BER \\
Gaussian Low Pass Filter [3] & 0.9780 & 0.0225 & 1 & 0 & 1 & 0 & 1 & 0 \\
Gaussian Low Pass Filter [5] & 0.9600 & 0.0420 & 1 & 0 & 1 & 0 & 1 & 0 \\
Gaussian Noise 0.001 & 0.9019 & 0.0967 & 1 & 0 & 1 & 0 & 1 & 0 \\
Gaussian Noise 0.005 & 0.7346 & 0.2715 & 1 & 0 & 1 & 0 & 1 & 0 \\
Median 3×3 & 0.9722 & 0.0283 & 1 & 0 & 1 & 0 & 1 & 0 \\
Median 5×5 & 0.7834 & 0.2813 & 0.7763 & 0.2266 & 0.9923 & 0.0078 & 0.8326 & 0.1689 \\
Salt \& Pepper Noise 0.001 & 0.9832 & 0.0166 & 1 & 0 & 1 & 0 & 1 & 0 \\
Salt \& Pepper Noise 0.01 & 0.8234 & 0.1719 & 0.9970 & 0.0029 & 1 & 0 & 1 & 0 \\
Speckle Noise 0.1 & 0.7681 & 0.2266 & 0.9941 & 0.0059 & 1 & 0 & 1 & 0 \\
Speckle Noise 0.4 & 0.6823 & 0.3223 & 0.8705 & 0.1250 & 1 & 0 & 1 & 0 \\
Poisson Noise & 0.8256 & 0.1729 & 1 & 0 & 1 & 0 & 1 & 0 \\
Adjust & 0.9950 & 0.0049 & 1 & 0 & 1 & 0 & 1 & 0 \\
Histogram Equalization & 0.9030 & 0.0957 & 1 & 0 & 1 & 0 & 1 & 0 \\
\hline
\end{tabular}

Table 3. Comparison of NC and BER Values under Geometrical Attacks, JPEG and JPEG2000

\begin{tabular}{cccccccccc}
\hline & \multicolumn{4}{c}{ Watermark 1 } & \multicolumn{4}{c}{ Watermark 2 } \\
\cline { 2 - 10 } Attacks & \multicolumn{2}{c}{ Ernawan [7] } & \multicolumn{1}{c}{ Proposed Scheme } & \multicolumn{2}{c}{ Ernawan [7] } & \multicolumn{2}{c}{ Proposed Scheme } \\
& NC & BER & NC & BER & NC & BER & NC & BER \\
Cropping rows off 25\% & 0.8100 & 0.1699 & 0.8388 & 0.1465 & 0.8393 & 0.1475 & 0.8252 & 0.1592 \\
Cropping column off 25\% & 0.8824 & 0.1094 & 0.9067 & 0.0879 & 0.8252 & 0.1592 & 0.8193 & 0.1641 \\
Rotation 8 & 0.5069 & 0.4922 & 0.4651 & 0.5146 & 0.4203 & 0.5186 & 0.4650 & 0.4971 \\
JPEG QF=50 & 0.8203 & 0.1729 & 0.9394 & 0.0586 & 0.8738 & 0.1270 & 0.8360 & 0.1582 \\
JPEG QF=90 & 1 & 0 & 1 & 0 & 1 & 0 & 1 & 0 \\
JPEG2000 CR=3 & 0.9970 & 0.0029 & 1 & 0 & 1 & 0 & 1 & 0 \\
JPEG2000 CR=5 & 0.8625 & 0.1533 & 1 & 0 & 1 & 0 & 1 & 0 \\
JPEG2000 CR=9 & 0.6794 & 0.3750 & 1 & 0 & 1 & 0 & 1 & 0 \\
\hline
\end{tabular}

The proposed multiple watermarking scheme provides an improvement in terms of NC and BER values for both extracted watermarks. Our scheme shows a strong resistance of extracting watermark under the Gaussian low pass filter, Gaussian noise, salt and pepper, speckle noise, adjust and histogram equalization. While, the proposed scheme produces less robust against median filter [5]. Our scheme shows a statistical improvement on the extracted first watermark than Ernawan's scheme [7] against cropping the image. While, the extracted second watermark needs to be further improved. Our scheme also shows superior robustness against JPEG and JPEG2000 compression. 


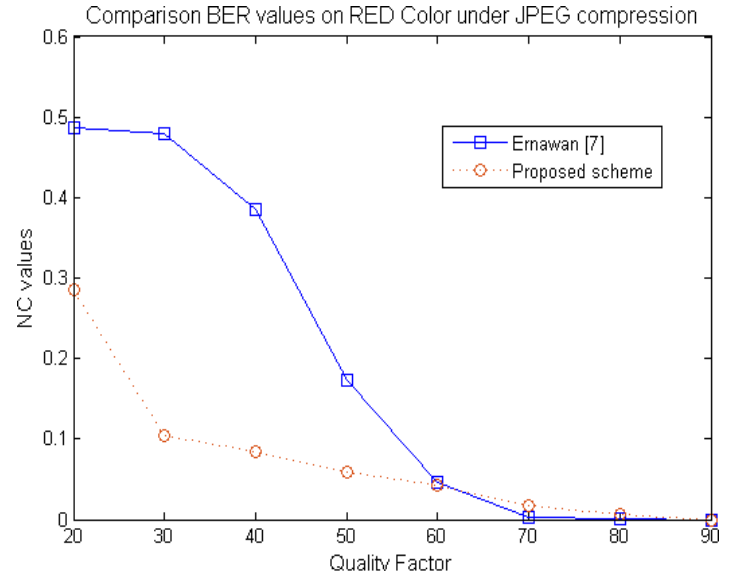

(a)

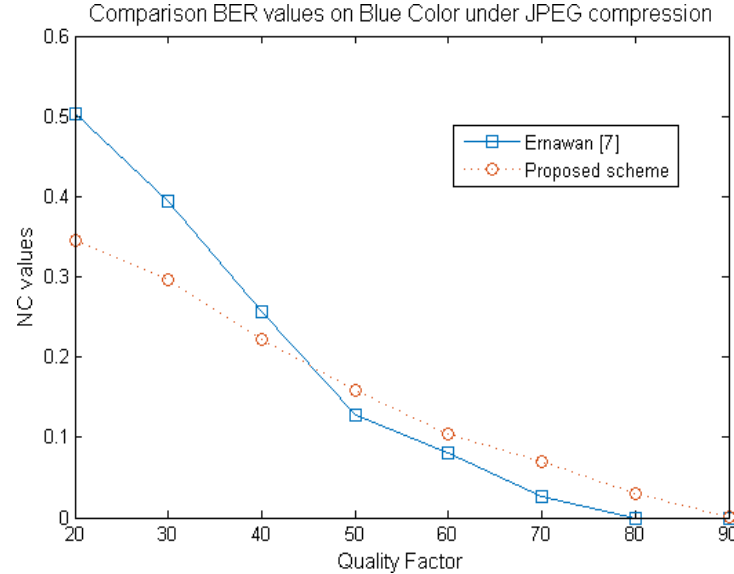

(b)

Figure 7. Comparison of BER values from Ernawan's scheme and the proposed scheme on (a) red color and (b) blue color

According to Figure 7, our scheme produces slightly greater BER values than Ernawan's scheme under JPEG compression with quality factor of 50, 60, 70, 80 and 90 in blue color. Whereas, the proposed scheme provides significant minimum BER values of the embedded watermark on a red color. Overall, the proposed scheme outperforms to the existing multiple watermarking technique. The visual perceptions of the extracted multiple watermarks from Ernawan's scheme and proposed scheme are shown in Table 4. The visual quality of the proposed scheme shows less distortion on the extracted watermark especially under noise addition and image filter. The extracted first watermark image of the proposed scheme provides higher visual quality than Ernawan's scheme against various attacks.

Table 4. The visual Watermark Recovery under Image Processing, Geometrical Attacks, JPEG and

\begin{tabular}{|c|c|c|c|c|}
\hline \multicolumn{5}{|c|}{ JPEG2000 } \\
\hline \multirow[b]{2}{*}{ Attacks } & \multicolumn{2}{|c|}{ Ernawan [7] } & \multicolumn{2}{|c|}{ Proposed Scheme } \\
\hline & Extracted & Extracted & $\begin{array}{c}\text { Extracted } \\
\text { Exarar }\end{array}$ & $\begin{array}{c}\text { Extracted } \\
\text { Sexare }\end{array}$ \\
\hline \multicolumn{5}{|c|}{ Gaussian Low Pass Filter [3] } \\
\hline \multicolumn{5}{|c|}{ Gaussian Noise 0.001} \\
\hline \multicolumn{5}{|l|}{ Median $3 \times 3$} \\
\hline \multicolumn{5}{|c|}{ Salt \& Pepper Noise 0.001} \\
\hline \multicolumn{5}{|c|}{ Speckle Noise 0.1} \\
\hline \multicolumn{5}{|l|}{ Adjust } \\
\hline \multicolumn{5}{|c|}{ Cropping rows off $25 \%$} \\
\hline \multicolumn{5}{|c|}{ Cropping column off $25 \%$} \\
\hline \multicolumn{5}{|l|}{ JPEG Q=50 } \\
\hline JPEG Q $=80$ & & & & \\
\hline
\end{tabular}




\section{CONCLUSION}

This paper presents multiple watermarking techniques using the optimal thresholds on red and blue colors. We select these colors due to the human visual system is more sensitive to green color. Each red and blue color is embedded by using different watermark images. Both watermark images are scrambled by Arnold transform before embedding watermarks occur. The human visual characteristics are utilized to identify less sensitive of selected blocks to human eyes. Selected blocks with minimum entropy values are embedded by examining the matrix U of DWT-SVD. We select two coefficients on matrix U for embedding the multiple watermark bits. This paper reveals the optimal thresholds for red and blue colors by evaluating the trade-off between SSIM and NC value against the JPEG quantization step. For robustness evaluation, our scheme is tested under various attacks. The experimental results show that our scheme achieves strong resistant under JPEG2000, filter and noise attacks. Our scheme produces a good visual quality of extracted both watermarks than existing techniques.

\section{ACKNOWLEDGEMENTS}

This work was supported by UMP Research Grant Scheme (RDU180358) from Universiti Malaysia Pahang, Malaysia.

\section{REFERENCES}

[1] L. Xiong, et al., “A Multiple Watermarking Scheme Based on Orthogonal Decomposition,” Multimedia Tools and Applications, vol. 75, pp. 5377-5395, 2016.

[2] G. Bhatnagar and Q.M. J. Wu, "A New Robust and Efficient Multiple Watermarking Scheme", Multimedia Tools Application, vol. 74, pp. 8421-8444, 2015

[3] X. Wei Li and I. Kwon Lee, "Robust Copyright Protection using Multiple Ownership Watermarks," Optics Express, vol. 23, no. 3, pp. 3035-3046, 2015

[4] N. M. Makbol, et al., "Block-Based Discrete Wavelet Transform-Singular Value Decomposition Image Watermarking Scheme using Human Visual System Characteristics," IET Image Processing, vol. 10, no. 1, pp. 34$52,2016$.

[5] S. Roy and A. K. Pal, "A Blind DCT Based Color Watermarking Algorithm for Embedding Multiple Watermarks," AEU - International Journal of Electronics and Communications, vol. 72, pp. 149-161, 2017.

[6] N. Mohananthini and G. Yamuna, "A Study of DWT-SVD Based Multiple Watermarking Scheme for Medical Images," International Journal of Network Security, vol. 17, no. 5, pp. 558-568, Sept 2015.

[7] F. Ernawan, et al., "A Blind Multiple Watermarks Based on Human Visual Characteristics," International Journal of Electrical and Computer Engineering (IJECE), vol. 8, no. 4, pp. 2578-2587, 2018.

[8] A. Zear, et al., "A Proposed Secure Multiple Watermarking Technique Based on DWT, DCT and SVD for Application in Medicine," Multimedia Tools Application, vol. 77, pp. 4863-4882, 2018.

[9] A. Kumar Singh, "Improved Hybrid Algorithm for Robust and Imperceptible Multiple Watermarking using Digital Images," Multimedia Tools Application, vol. 76, pp. 8881-8900, 2017.

[10] H. -T. Hu and L. -Y. Hsu, "Exploring DWT-SVD-DCT Feature Parameters for Robust Multiple Watermarking against JPEG and JPEG2000 Compression," Computers and Electrical Engineering, vol. 41, pp. 52-63, 2015.

[11] X.L. Liu, et al., "Blind Dual Watermarking for Color Images' Authentication and Copyright Protection," IEEE Transactions on Circuits and Systems for Video Technology, vol. 28, no. 5, pp. 1047-1055, May 2018.

[12] N. Mohananthini and G. Yamuna, "Comparison of Multiple Watermarking Techniques using Genetic Algorithms," Journal of Electrical Systems and Information Technology, vol. 33, no. 1, pp. 68-80, 2016.

[13] Z. Zhang, et al., "Image Watermarking Scheme Based on Arnold Transform and DWT-DCT-SVD," IEEE 13th International Conference on Signal Processing (ICSP), 2016, pp. 805-810.

[14] S. Fazli and M. Moeini, "A Robust Image Watermarking Method Based on DWT, DCT and SVD using A New Technique for Correction of Main Geometric Attacks," Optik, vol. 127, no. 2, pp. 964-972, 2016.

[15] F. Ernawan and M.N. Kabir, "A Block-Based RDWT-SVD Image Watermarking Method Using Human Visual System Characteristics," The Visual Computer, pp. 1-19, 2018.

[16] M. R. Abuturab, "Multiple Color-Image Fusion and Watermarking Based on Optical Interference and Wavelet Transform," Optics and Lasers in Engineering, vol. 89, pp. 47-58, 2017.

[17] F. Ernawan and D. Ariatmanto, "Image Watermarking Based on Integer Wavelet Transform-Singular Value Decomposition with Variance Pixels," International Journal of Electrical and Computer Engineering (IJECE), vol. 9, no. 3, pp. 2185-2195, 2019.

[18] Wang J, et al., "Hybrid Multiplicative Multi-Watermarking in DWT Domain," Multidimensional Systems and Signal Processing, vol. 28, no. 2, pp. 617-636, 2017.

[19] F. Ernawan, et al., "An Improved Imperceptibility and Robustness of 4x4 DCT-SVD Image Watermarking Using Modified Entropy," Journal of Telecommunication, Electronic and Computer Engineering, vol. 9, no. 2-7, pp. 111$116,2017$. 
[20] M. Ali, et al., "A Robust Image Watermarking Technique using SVD and Differential Evolution in DCT Domain," Optik, vol. 125 , pp. 428-434, 2014.

[21] F Ernawan, MN Kabir, "A blind watermarking technique using redundant wavelet transform for copyright protection," 14th International Colloquium on Signal Processing \& Its Applications (CSPA), pp. 221-226, 2018.

[22] F. Ernawan, et al., "Block-based Tchebichef image watermarking scheme using psychovisual threshold," International Conference on Science and Technology-Computer (ICST), pp. 6-10, 2016.

[23] F. Ernawan, "Tchebichef image watermarking along the edge using YCoCg-R color space for copyright protection,"

International Journal of Electrical and Computer Engineering (IJECE), vol. 9, no. 3, pp. 1850-1860, 2019.

[24] F. Ernawan and M. N. Kabir. "A Robust Image Watermarking Technique with An Optimal DCT-Psychovisual Threshold,"

IEEE Access, vol. 6, pp. 20464-20480, 2018.

[25] F. Ernawan, "Robust Image Watermarking Based on Psychovisual Threshold," Journal of ICT Research and Applications,

vol. 10, no. 3, pp. 228-242, 2016.

\section{BIOGRAPHIES OF AUTHORS}

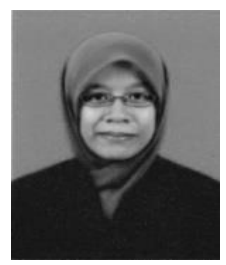

Noruhida Binti Alias was born in Muadzam Shah, Pahang, in 1993. She is currently a postgraduate student at the Faculty of Computer Systems \& Software Engineering, Universiti Malaysia Pahang. She received the bachelor degree in Computer Science (Graphic \& Multimedia Technology), Universiti Malaysia Pahang in 2016. Her research interests in digital watermarking.

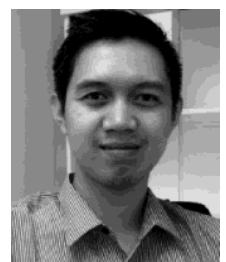

Ferda Ernawan was born in Semarang, Central Java, Indonesia, in 1988. He received the master's degree in software engineering and intelligence and the Ph.D. degree in image processing from the Faculty of Information and Communication Technology, Universiti Teknikal Malaysia Melaka in 2011 and 2014, respectively. He is currently a Senior Lecturer with the Faculty of Computer Systems \& Software Engineering, Universiti Malaysia Pahang. His research interests include image compression, digital watermarking and steganography. 